\section{Mathematical Practices}

Essays in Philosophy and History of Mathematics Brussels, Belgium

26 - 28 March 2007

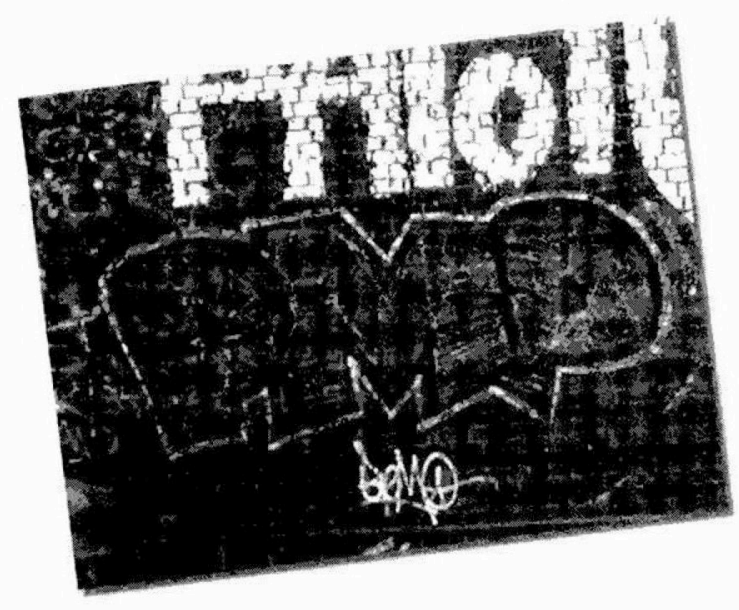

edited by

Bart Van Kerkhove

Vrije Universiteit Brussel, Belgium

\section{No world Scientific}

AN ENHANCED ARGUMENT FOR INNATE

ELEMENTARY GEOMETRIC KNOWLEDGE AND ITS PHILOSOPHICAL IMPLICATIONS

\author{
HELEN DE CRUZ \\ Centre for Logic and Analytical Philosophy \\ University of Leuven, Belgium \\ -E-mail: Helen.DeCruz@hiw,kuleuven.be \\ http://uruw.hiw.kuleuven.be/ned/onderzoek/claw.php
}

\section{Introduction}

The idea that geometry derives from innate knowledge has an ancient history in philosophy. As chronicled by Plato ${ }^{1}$ in Meno, ca. 380 B.C., Socrates probed the geometric intuitions of an uneducated slave boy in a Greek household, leading him, through a series of questions, to discover relationships between the areas of squares drawn in the sand. He concluded that the slave's soul must have always possessed this knowledge, and that our learning of geometric concepts is actually recollecting (anamnesis) what we have always known as immortal souls. Descartes ${ }^{2}$ (AT VI 135-137) wondered how a blind person is able to learn spatial relationships by touch, and extended this problem to sighted people who learn to orient themselves in space by sight. He suggested that this is possible through une géométrie naturelle, an innate geometry (Descartes, ${ }^{2}$ AT VI 137-138). The most detailed philosophical argument for the innateness of geometric intuitions comes from Kant's argument from geometry, which argues that our geometric knowledge derives from an a priori intuition of space.

This paper aims to look at this longstanding philosophical issue through the lens of cognitive science. I will investigate whether empirical data from studies on animal cognition, developmental psychology and anthropology warrant an enhanced version of the argument from geometry. In Section 2, I briefly discuss the argument from geometry and propose an interpretation of this argument which can be pitted against the evidence from cognitive science and the history of mathematics. Section 3 considers evidence for 
spatial intuitions in animals, young children and toddlers, and looks at the neurobiological underpinnings of this ability. Section 4 examines anthropological and historical data for continuities and discontinuities between our spatial intuitions and formal geometry. Section 5 assesses whether these data support the enhanced argument from geometry and provides some suggestions for future research.

\section{The argument from geometry}

\subsection{Kant's original argument from geometry}

The argument from geometry appears in Kant's Critique of pure reason (1781). ${ }^{3}$ First, in the section On space, metaphysical exposition of this conception, Kant stresses that the original representation of space is an a priori intuition (Kant, ${ }^{3}$ A25/B40). He specifies that our intuition of space does not and cannot be derived from outward experience:

Space is not an empirical concept that has been drawn from outer experiences. For in order for certain sensations to be related to something outside me (i.e., to something in another place in space from that in which I find myself), thus in order for me to represent them as outside one another, thus not merely as different but as in different places, the representation of space must already be their ground. Thus the representation of space cannot be obtained from the relations of outer appearance through experience, but this outer experience is itself first possible only through this representation (Kant, ${ }^{3}$ B38).

Then, in the paragraph entitled Transcendental exposition of the concept of space, Kant argues that it is precisely this intuition of space that enables us to develop geometry as a scientific discipline:

Geometry is a science that determines the properties of space synthetically and yet a priori. What then must the representation of space be for such a cognition of it to be possible? It must originally be intuition; for from a mere concept no propositions can be drawn that go beyond the concept, which, however, happens in geometry. But this intuition must be encountered in us a priori, i.e., prior to all perception of an object, thus it must be pure, not empirical intuition. For geometrical propositions are all apodictic, i.e., combined with consciousness of their necessity, e.g., space has only three dimensions; but such propositions cannot be empirical or judgments of experience, nor inferred from them. Now how can an outer intuition inhabit the mind that precedes the objects themselves, and in which the concept of the latter can be determined a priori? Obviously not otherwise than insofar as it has its seat merely in the subject, as its formal constitution for being affected by objects and thereby acquiring immediate representation, i.e., intuition, of them, thus only as the form of outer sense in general. Thus our explanation alone makes the possibility of geometry as a synthetic a priori cognition comprehensible (Kant, ${ }^{3} \mathrm{~B} 41$, emphasis added).

Philosophers of mathematics have generally taken this passage as the unreasonable claim that geometry necessarily reflects eternal, unchanging and universal intuitions of space. Indeed, many agree that the development of non-Euclidean geometries refuted Kant's argument altogether (e.g., Hersh, ${ }^{4}$ pp. 131-2). However, Shabel ${ }^{5}$ has persuasively argued for an alternative weaker interpretation for the argument from geometry: it does not analyze geometric cognition in order to establish that we have an a priori intuition of space. Rather, it establishes that geometric cognition itself develops out of a pure intuition of space. This intuition is offered both as the actual source of our cognition of the first principles of geometry and as a basis for culturally transmitted geometric concepts. This does not exclude the cultural evolution of geometric principles that do not correspond to, or even violate, these intuitions. Rather, it amounts to the modest claim that without a priori intuitions of space, geometry of any kind would be impossible. They admit the possibility of geometry, as Kant puts it, rather than any particular formal geometric system. The a priori concept of space is underdetermined, but it governs our capacity to form intuitions of particular finite spatial regions $\left(\right.$ Shabel $\left.^{6}\right)$.

\subsection{An enhanced argument from geometry}

It is not my intention of reformulating or explicating Kant's argument, as this has already been aptly done by other scholars. However, I use Kant as my starting point for gauging the role of spatial intuitions in geometrical knowledge as he provides the most detailed formulation of the a priori basis of spatial cognition and geometry. Based on this careful re-examination of the argument from geometry, I can now formulate an enhanced version of this argument:

Humans possess an innate intuition of space. 
This pure intuition of space constrains and governs the development of formal geometry.

In recent years, a growing number of philosophers of mind have turned to cognitive science (e.g., De Cruz, ${ }^{7}$ Decock, ${ }^{8}$ Margolis and Laurence ${ }^{9}$ ) to examine how people acquire concepts and other forms of abstract knowledge. Working within the framework of naturalized epistemology $\left(Q u i n e^{10}\right.$ ), they use findings from empirical science to examine the real-world conditions under which epistemic agents acquire knowledge within the scope and limits of their cognitive capacities. Within the framework of naturalized epistemology, the argument from geometry thus becomes a statement whose truth value depends to an important extent on cognitive science for the answer to the following questions: is there any evidence for innate intuitions of space, and do these intuitions play a role in geometry? Fortunately, we are in an excellent evidentiary position to do this. Investigations of the cognitive capacities that underlie our knowledge of space have a rich and diverse history, especially given the fact that cognitive science is such a young discipline. Since the 1960 s, a large body of evidence has accumulated on how animals navigate and how they represent space. Since the 1970 s, neuroscientists have been investigating how space is represented at the neural level. About a decade later, developmental psychologists began to investigate how toddlers and young children represent space. Recent work has focused on the question in how far human spatial cognition is continuous with nonhuman animal representations of space, which can help us evaluate the claim that humans possess an innate intuition of space. The second part of the enhanced argument from geometry can be evaluated as follows: is there evidence that geometry depends on this innate capacity for reasoning about space? Evidence in favor of this claim should come from continuities between intuitive notions of space and formal geometry observed in the history of mathematics and from other cultures.

\section{The cognitive basis of spatial cognition}

\subsection{Evidence from studies of animal spatial cognition}

From an evolutionary perspective, there are good reasons to expect that animals possess a priori intuitions of space. All animals must be able to find their way back to particular locations like food-sources, hives, nests and caches. Over the past 50 years, studies on animal navigation indicate that animals possess a wide variety of specialized mechanisms that help them to navigate. Some of these abilities are highly specialized, such as the homing pigeon's (Columba livia) sensitivity to the Earth's magnetic field (Mora et al. ${ }^{11}$ ), or the indigo bunting's (Passerina cyanea) ability to use trigonometric relationships between clusters of stars to orient itself during migration $\left(\mathrm{Emlen}^{12}\right)$. Of course, as one reviewer to this paper has aptly remarked, cognitive adaptations for navigation do not automatically entail sophisticated spatial skills. Some species' navigational skills can be explained by an efficient interaction between their sensori-motor capacities and properties of the environment, such as Pharaoh's ants' (Monomorium pharaonis) ability to use pheromone trails to find their way back from a food source to the nest (Jackson et al. ${ }^{13}$ ). Another simple solution to the problem of navigation, found in insects such as ants and bees, is to store mental snapshots of landmarks encountered along paths from the nest to a food-source. However, this solution is relatively inflexible and demands much memory resources - thousands of images need to be stored as animals need to make multiple snapshots of a particular landmark from different vantage points so as to recognize it from multiple angles (Judd and Collett $\left.^{14}\right)$. For animals with a wide and variable home range, it may be useful to represent spatial properties on a more abstract level. Because geometric relationships are salient, we can expect that many species have evolved sensitivity to such cues.

Carefully controlled experiments have assessed whether animals can make high-level geometric representations. One research program has focused on Clark's nutcrackers (Nucifraga columbiana, a species of corvid). These food-caching birds can conceptualize the halfway point between two landmarks. Kamil and Jones ${ }^{15}$ trained Clark's nutcrackers to find a partially buried seed halfway between two plastic pipes. On the test trials, the seeds were entirely buried. The birds were presented with new distances between the pipes to test their ability to generalize the geometric relationship between the seed and the two landmarks. They readily learned to bisect the inter landmark distance: they correctly found the halfway point when the landmarks were presented with new distances between them. The geometric sense of the birds is quite abstract, since alteration of the height of one of the landmarks did not affect their success at finding the seed. Simpler explanations were experimentally ruled out, e.g., finding the seeds by smell or by cues other than geometry. Moreover, the birds were more accurate in finding the line connecting the landmarks than in locating the correct position on the line. This suggests that they make two separate decisions (Kamil and Jones ${ }^{16}$ ): finding the line connecting the landmarks, and subsequently determining the halfway point on it. Clark's nutcrackers 
store up to 33,000 pine seeds in thousands of cache sites during the autumn months. The seeds constitute the bulk of their winter diet, and are fed to their nestlings in spring (Gould-Beierle and Kamil ${ }^{17}$ ). Considering these selective pressures, it is not surprising that these corvids have evolved geometric abilities to retrieve previously stored seeds.

Cheng ${ }^{18}$ has experimentally shown that rats rely exclusively on geometric cues (the shape of a room) to reorient themselves in order to find food. Hungry rats explored a room with partially buried bits of food. After the food was fully buried, they were reintroduced. Although the animals were provided with a wealth of nongeometric information (here termed featural cues), such as distinctive odors and relative brightness of the walls, they apparently exclusively relied on one clue only, the shape of the room. The rats betrayed their search methods by looking with high frequency for the food at its true location as well as its geometric equivalent (a mistake termed the rotational error). For example, the rats would search in the two corners that are located to the left of the short walls, which are geometrically indistinguishable, as shown in Fig. 1. Subsequent studies revealed that the rats were indeed able to discriminate nongeometric cues, but that they were somehow unable to use them in remembering locations. These findings led Gallistel ${ }^{19}$ (p. 172) to propose that rats have an innate geometry module, which is informationally encapsulated, meaning that it does not have access to nongeometric information. While the claim that intuitive geometry is informationally encapsulated remains controversial (see Cheng and Newcombe, ${ }^{20}$ for a review), the idea that many species have specialized cognitive mechanisms to deal with the geometric properties of their environment has become widely accepted.

Not all species place a similar premium on geometry. Some species, such as chickens, goldfish and lizards can use both featural cues and geometry to orient themselves. Chicks (Gallus gallus domesticus) can use geometric cues to find food in a rectangular room; they betray their search method by the rotational error. Once the walls are visually distinguishable, they can solve the task almost perfectly. When featural and geometric information provide contradictory cues, they rely mostly on features (Vallortigara et al. ${ }^{21}$ ). Goldfish (Carassius auratus), too, encode both featural and geometric information; they even use geometry when it is not strictly necessary to solve the task (Vargas et al. ${ }^{22}$ ). The whiptail lizard (Cnemidophorus inornatus) uses both featural cues and geometry, but it is more accurate when it relies on geometry (Day et al. ${ }^{23}$ ). In sum, there is a considerable variation between species in the relative importance of featural and geometric

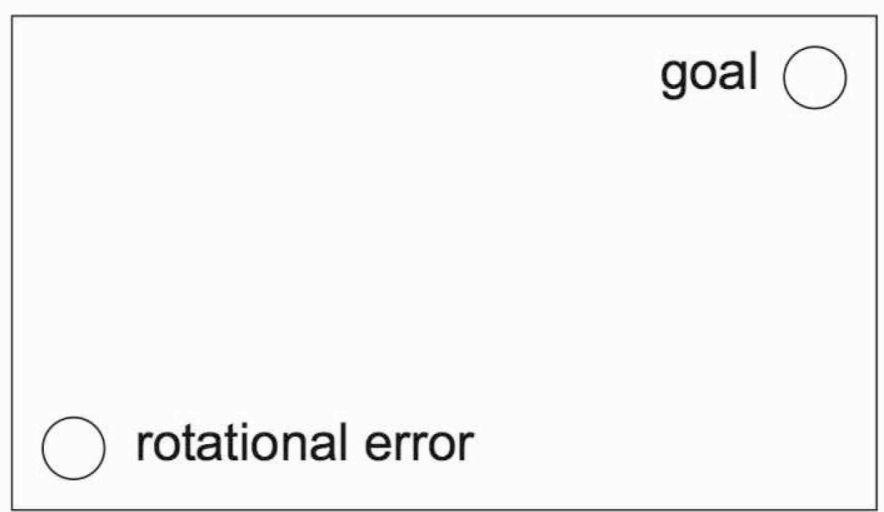

Figure 1. Schematic representation of the geometric effect in reorientation in a rectangular room

cues when navigating. As we shall see in the next section, developmental psychological studies indicate that humans are also capable of exploiting both kinds of information, but interestingly, our ability to derive geometric relationships emerges earlier in development than our ability to use featural cues.

\subsection{Evidence from developmental psychology}

Replicating Cheng's experiment, Hermer and Spelke ${ }^{24,25}$ demonstrated that 18- to 24-month-olds use geometric cues to look for a hidden toy: disoriented toddlers reliably looked in the geometrically appropriate corners of a rectangular room. Like rats, they were utterly incapable to orient themselves by use of nongeometric cues, such as the color of a wall or colorful boxes close to the hidden toy. Even when the experimenter pointed out these useful landmarks, the toddlers failed to make use of them (Hermer and Spelke, ${ }^{24}$ p. 58). Between five to seven years of age, children gradually exhibit more flexible reorientation behavior, paying attention to both landmarks and geometric relationships (Hermer-Vazquez et al. ${ }^{26}$ ). In another study, Haun et al. ${ }^{27}$ found that prelinguistic infants of 12 months prefer geometric over featural cues, and that this preference reverses at about three years of age. They presented infants, three-year-olds and four species of nonhuman apes (chimpanzee, bonobo, orang-utan and gorilla) with a simple object-search task, in which a reward was hidden under one of three containers, each of which looked distinctly different. The setup was then occluded, and two of the containers were switched. In the feature condi- 
tion, the reward moved with its distinctive container, whereas in the place condition, the reward remained in its original place but came under a different container. Both infants and nonhuman apes performed better in the place condition, indicating that they exclusively relied on geometric cues to obtain the reward. In contrast, three-year-olds did better in the feature condition. This research indicates that geometric cognition arises earlier in child development than featural spatial cognition. Since the spontaneous use of geometric cues emerges prior to language acquisition and is similar to that of our closest living relatives, it seems very plausible that geometric cognition arises prior to experience.

A detailed case-study of a two and a half-year-old blind child (Landau et al. ${ }^{28}$ ) shows that these early intuitions of space do not even require vision for their development. The girl was taken along several paths connecting four landmarks in a room, as is shown in Fig. 2 (dashed lines). The landmarks were easily distinguishable by touch, such as a table and a heap of pillows. During the test, she was asked to move directly between objects on paths she had never taken (solid lines). For example, during training, the child was guided to walk from her mother $(M)$ to the heap of pillows $(P)$, and back, and next from her mother $(M)$ to the basket of toys $(B)$. From there, she was asked "can you go to the pillows", a path she had not walked before. She spontaneously took the correct path $(B)-(P)$. The girl's accuracy in approaching the targets was striking; she even adjusted her movements as she went towards the targets, with 11 successful trials out of 12. A control experiment with blindfolded three-year-olds and adults revealed similar levels of success. To be able to deduce the correct path between two objects after moving to each of them from a third point, one must be able to derive angular relationships between previous routes, and the length of both routes. From this information, one can derive new angular relationships: the angular direction from one object to the other. Angle and distance are properties that are both conserved in metric geometries such as Euclidean geometry, but not in non-metric geometries, such as topology and projective geometry (the latter conserves only angles). Interestingly, the axioms of Euclidean geometry suffice to solve the aforementioned task. Landau et al. ${ }^{28}$ therefore concluded that some principles of Euclidean geometry are known from an early age on, and that sight is not required to learn about them

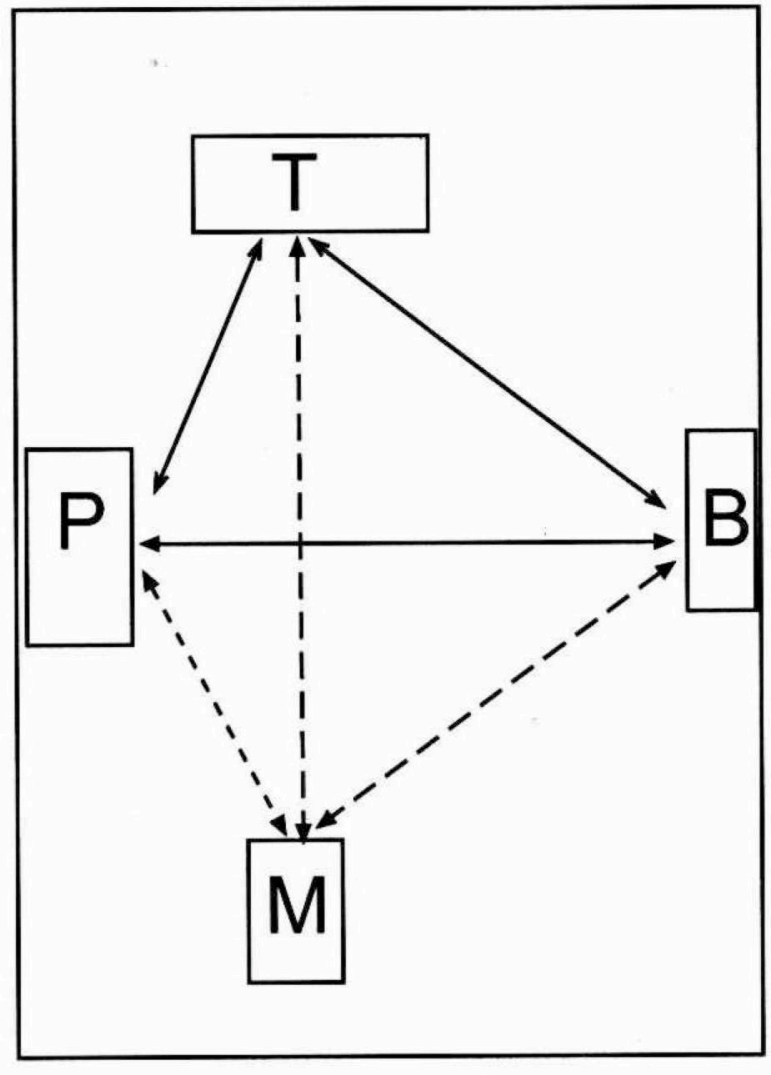
Figure 2. Room layout for spatial inference experiment. Dashed lines indicate paths
taken during training, solid lines indicate test paths, redrawn from Landau et al., ${ }^{28}$ p. 1275 , fig. 1)

\subsection{Evidence from neuroscience}

Does the ability of humans and other animals to derive geometric relationships from the environment depend on a specialized neural circuitry? In the 1970 s, neurobiologists O'Keefe and Dostrovsky ${ }^{29}$ discovered that individual neurons in the rodent hippocampus increase their firing rates when the animal traverses specific regions of its surroundings. Each of these place cells has its own place field, typically covering about 10 to 20 percent of the current environment, only firing when the animal is located somewhere within its place field, and being silent when it is outside of it. In this way, place cells together constitute a kind of cognitive map. Lever et al. ${ }^{30}$ found that the firing patterns of place cells of a rat placed in rooms in which the walls 
form a square or a circle increasingly diverged as the animal got familiar with the two geometric configurations. In other words, there were individual cells that fired preferentially responding to the circle, and those that fired only when the rat was in a square-shaped room. When the rats were placed in novel enclosures, the distinct firing patterns were immediately transferred to novel enclosures of the same shape. These patterns persisted after a delay of several weeks. This work suggests that place cells can represent the geometry of an environment at an abstract level, and possibly, that some place cells may be specifically attuned to geometry. Place cells do not simply code visual properties: the place cells of early blind rats, which have never seen their environment, respond in the same way as place cells of sighted rats (Save et al. ${ }^{31}$ ).

Hafting et al. ${ }^{32}$ found that neurons in the entorhinal cortex of freely roaming rats also respond to spatial properties: if plotted on a map of the environment, the firing locations of the neurons form a triangular grid, resulting in a triangular lattice. Like place cells, these grid cells do not depend on visual input: once the rat is familiar with a particular enclosure, they keep firing in the same pattern when the animal roams in total darkness (Hafting et al., ${ }^{32}$ p. 803). While place cells can be conceptualized as a map, the grid cells' role is to provide a coordinate system, not unlike the overlaid grids of a map - except that the unit of the grid is not a square but an equilateral triangle. ${ }^{a}$ When a rat occupies a particular location, the grid cell that represents this position will be very active, together with its neighboring cells. As the rat moves, so does this field of activation, comparable to a moving 'you are here' sign on a map. To maintain this mapping between the rat's real position and the cognitive map provided by the place cells, specialized grid cells encode information about head-direction, position, and even the speed with which the animal moves (Sargolini et al. ${ }^{33}$ ).

a There may be good reasons why the lattice is triangular rather than simply square. Mathematical models of percolation can model the connectivity of a lattice. Each site in a lattice (in this case, each triangle) can be part of a cluster (i.e., a group of neighboring sites that have the same state, namely occupied or empty) with probability $p$. If $p$ is small, there are many disconnected clusters; if $p$ is large, however, there are fewer large clusters. If $p \approx 1$, most of the occupied cells will form one large cluster, extending from one end of the lattice to the other. If grid cells are to be useful in navigation, connectivity between them may not be too high (as too many cells would fire and the animal would become confused about its actual location), nor too low (as the animal would be unable to figure out its trajectory). The $p$ value for an individual site to be part of a cluster in a triangular lattice is exactly $\frac{1}{2}$ (I thank Lesley De Cruz for pointing this out to me). a triangular lattice is exactly $\frac{1}{2}$ (I thank Lesley De Cruz for pointing this out to me).
It would be interesting to examine whether this connectivity is optimal for navigational purposes.
The neurobiology of navigation reflects the two main strategies for keeping track seen in animals: a geometric strategy, which depends heavily on relationships between features of the environment, and path integration, in which animals use self-generated motion cues to update a vector-based representation of distance and direction from a fixed reference point (Witter and Moser $^{34}$ ).
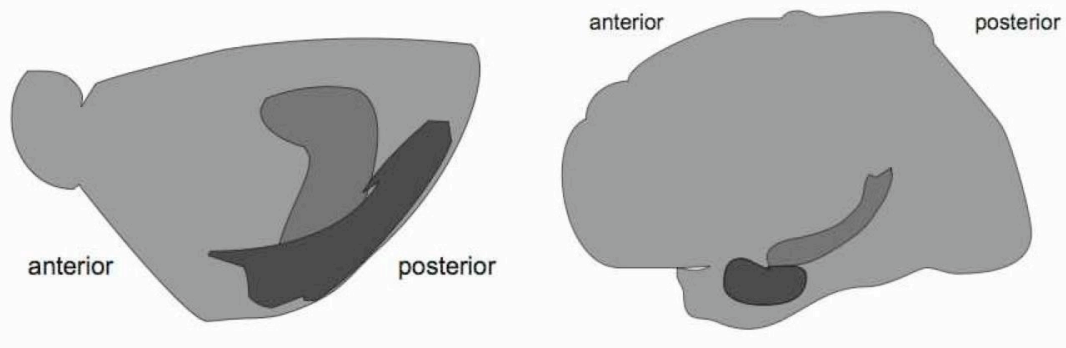

entorhinal cortex (includes parahippocampal area)

hippocampus

Figure 3. Areas in the rat brain (left) and the human brain (right) dealing with the representation of space (only left hemisphere shown; brains not to scale)

Because recording individual neurons requires invasive brain surgery, it has long been unclear whether the human brain contains similar spacesensitive neurons. Ekstrom et al..$^{35}$ recorded single neurons in seven epileptic patients prior to brain surgery, while they navigated in a virtual world. They found neurons in the hippocampus and parahippocampal region (which encompasses the entorhinal cortex) that responded to spatial properties (Fig. 3). Many cells preferentially responded to a single landmark. Most neurons in the parahippocampus were view-independent: they fired regardless of the angle or view from which the subject saw the landmark. In this way, the parahippocampus extracts allocentric spatial information from the salient visual landmarks. It provides us with the basic geometric layout of the environment. This study is in accordance with earlier neuroimaging studies (e.g., Epstein and Kanwisher ${ }^{36}$ ) which indicate that the geometric layout of an environment is constructed in the parahippocampal place area. Patients with focal brain damage to this area have an impaired ability to process geometric information: though they are able to produce accurate maps of places they knew before the brain lesion occurred, they are unable 
to produce accurate maps of places they have encountered since. However, their perceptual abilities for nongeometric objects remained largely intact $\left(\right.$ Epstein et al. $\left.{ }^{37}\right)$. This indicates a double dissociation between vision and geometry. In sum, neurophysiological recordings suggest that human reasoning about geometric relationships rests on specialized neural circuitry, which deals with a higher-order representation of space.

\subsection{Evolutionary origins of human spatial cognition}

Given that navigation is a universal problem for animals, it is unsurprising that all species examined for it possess the ability to attend to geometric relationships between landmarks. Next to this, human adults and a variety of other species also attend to nongeometric (featural) information when they navigate. Given that toddlers, young children and nonhuman apes attend primarily to geometry when dealing with search tasks, it seems likely that geometric intuitions are a stable and universal part of human cognition. Taken together, this evidence is in favor of the first part of my reformulation of Kant's argument from geometry, namely that humans possess an innate intuition of space.

\section{Does geometry develop out of a pure intuition of space?}

This does not automatically entail the second part of the enhanced argument from geometry, namely that geometry develops from this pure intuition of space. After all, the gap between our geometric intuitions, impressive as they are, and geometry as a formal science is immense. To examine whether our innate intuition of space constrains and governs geometry, we need not examine geometry in its current form, but rather its historical roots. Historically, geometry has developed to provide formal and standardized solutions to problems frequently encountered in land surveying, navigation, engineering and architecture, such as how to square surface areas. This presupposes the ability to reason about abstract spatial relationships between objects, regardless of their features. Without this a priori intuition, it might never occur to us to calculate the ratio of a diagonal of a square to its side as $\sqrt{2}-$ any square, regardless of its actual size or features.

\subsection{Evidence from anthropology}

Cultures display a considerable variation in how space is represented, and this variation is expressed in linguistic differences. For example, Dutch and
English speakers use mainly relative, viewpoint-dependent (and thus egocentric) spatial terms, such as 'the ball lies to the left of the tree'. Speakers of several indigenous Australian, Papua, and !Kung languages use absolute terms, e.g., 'the water is in the northern well'. Experimental evidence indicates that spatial strategies are influenced by these cultural or linguistic preferences: !Kung children do better in search tasks where objects maintain their position in relation to the surrounding area, whereas Dutch children perform better in tasks where objects maintain their position in relation to themselves (Haun et al. ${ }^{38}$ ). This cultural variation indicates that our intuitive notions of space are underdetermined; their development is subject to cultural variation.

How much, then, of our geometric intuitions are prespecified? Although the empirical evidence on this point needs to be further developed, evidence from anthropology and the history of mathematics provides tentative support for the second part of the enhanced argument from geometry, namely that we do not just have an innate intuition of space, but that this intuition can also serve as a basis for the development of formal geometry. Through an anthropological case study, Dehaene et al. ${ }^{39}$ argue that some properties of Euclidean geometry may be part of our intuitive knowledge. The Mundurukú, an Amazonian culture, do not possess maps, little (if any) schooling, no compasses, nor do they possess an intricate geometric vocabulary. In their study, Dehaene et al. ${ }^{39}$ asked Mundurukú participants to choose among arrays of six images the 'ugly' or 'weird' one. In each array, five pictures instantiated a concept from Euclidean geometry (e.g., parallelism, points, lines and symmetry), while one violated it. The figures were designed to minimize cues other than the desired conceptual relation that could identify the target figure (i.e., the odd one out). All participants performed significantly above chance level; there was no difference between children and adults. Fig. 4 shows samples of the test arrays, with the deviant figure marked in grey. The percentage of participants choosing the correct intruder is shown above each array; results higher than 35 percent are statistically significant. As can be seen in Fig. 4a, subjects were able to discriminate curves from straight lines, see Euclid's fourth definition. They could see whether a point was aligned with a straight line or not, an assumption implicit in Euclid's Elements, often used in his propositions (Fig. 4b). They detected parallel lines in a collection of secant lines and vice versa, see Euclid's fifth postulate (Fig. 4c), as well as a right angle in a collection of non-right angles and vice versa, see Euclid's fourth postulate (Fig. 4d). The Mundurukú provide an interesting example because, 
like many other cultures, they do not possess a formalized geometry. Nevertheless, they spontaneously detected Euclidean-like geometric principles.

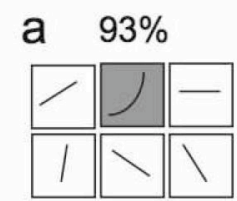

straight line

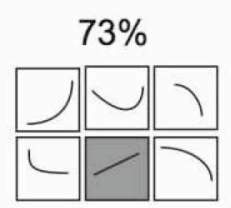

curve

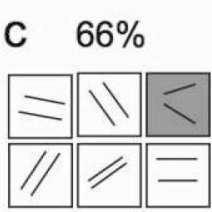

parallel lines
$80 \%$

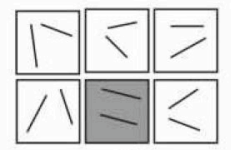

secant lines

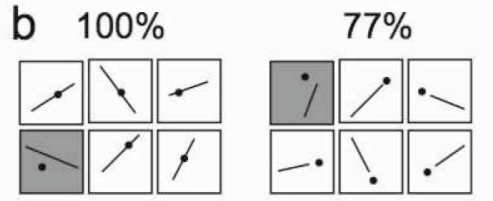

alignment of points in lines

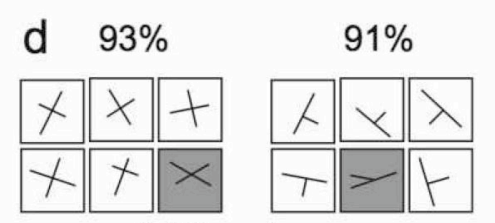

right angle
Figure 4. Performance of Mundurukú participants in a multiple-choice test of some core concepts of geometry, adapted from Dehaene et al., ${ }^{39}$ p. 382 , fig. 1)

To claim that these principles are universal human cognitive features needs further support. For example, one could replicate this study in different cultures or with young children. In this way, one could examine whether 'folk geometry' has features that are invariant across culture, in a similar way as the investigations on folk biology (e.g., $\operatorname{Atran}^{40}$ ) or folk psychology (e.g., Callaghan et al. ${ }^{41}$ ). If the Mundurukú results can be replicated in other cultures, this could provide compelling support for the intuitiveness of Euclidean geometry.

\subsection{The success and persistence of Euclidean geometry}

For centuries, Euclid's parallel postulate ${ }^{\mathrm{b}}$ was taken as intuitively true, albeit less self-evident than the other four. Mathematicians felt it had to be proved as a theorem from the other four. Although many tried, none succeeded. In 1733, the Italian Giovanni Gerolamo Saccheri unintentionally invented a new viable hyperbolic geometry while trying to prove Euclidean

\footnotetext{
${ }^{b}$ If two lines intersect a third in such a way that the sum of the inner angles on one side is less than two right angles, then the two lines must inevitably intersect each other on that side if extended far enough.
}

geometry in his Euclides ab omni naevo vindicatus (Euclid freed from all flaws). Less known is that Muslim mathematicians, more than six centuries earlier, also wrote commentaries on the Elements, and that they also devoted special attention to the parallel postulate (Kanani, ${ }^{42}$ pp. $\left.316-9\right)$. No one doubted its validity, but many felt that it could not be accepted without proof. Ibn al-Haytham (865-901) introduced the concept of motion into geometry. His proof of the parallel postulate goes as follows: given point $P$ not on line $L_{1}$, the shortest distance between them is the line $P S$, which goes through point $P$ and perpendicular to line $L_{1}$. If we start moving $P S$ along the line $L_{1}$, a new line $L_{2}$ will be constructed. $L_{2}$ is parallel to $L_{1}$ since the distance between $P$ and $L_{1}$ remains unchanged. Omar Khayyám (1048-1131) objected to this introduction of motion in geometry. To him, motion was an attribute of matter; it should therefore not be introduced into the immaterial world of mathematics. In his Explanation of the difficulties in Euclid's postulates, written around 1100, he used an indirect proof, arguing that the assumption that the fifth postulate was false would lead to a contradiction. His proof is summarized in Fig. 5. In the course of his attempt to prove the parallel postulate, Khayyám was led to posit some non-Euclidean theorems. He constructed two line segments $A C$ and $B D$, perpendicular to $A B$, to construct $A B C D$. The angle $A C D$ is equal to angle $B D C$. He then examined three possible cases: (a) angles $A C D$ and $B D C$ are each less than $90^{\circ}$; (b) angles $A C D$ and $B D C$ are each larger than $90^{\circ}$; (c) angles $A C D$ and $B D C$ are each equal to $90^{\circ}$. The KhayyámSaccheri quadrilateral (the grey area in Fig. 5), composed of line segment $C D$, and two equal legs standing at right angles to it, $C K$ and $D H$, has oblique angles at $K H$ in cases (a) and (b). However, he never envisaged the possibility of a non-Euclidean geometry, because he was convinced that Euclidian geometry was the only true one (Kanani, ${ }^{42}$ p. 319$)$. For the same reason, Saccheri failed to recognize the significance of his discoveries.

Around 1830, attempts to prove the parallel postulate culminated in the purportedly independent invention of hyperbolic geometry by Nicolai Lobatchevsky, János Bolyai and Karl Friedrich Gauss. Hyperbolic geometry fulfils all of Euclid's postulates and common notions, except that the fifth postulate does not hold, as there are at least two distinct lines through point $P$ which do not intersect line $L$. The realization that Euclidean geometry was not the only possible way to look at spatial relationships has enabled mathematicians to disregard the semantic content that was traditionally associated with some mathematical concepts. Traditionally, geometry had an obvious semantic component: spatial relationships. But today, primitive 
$\mathrm{K}$

G

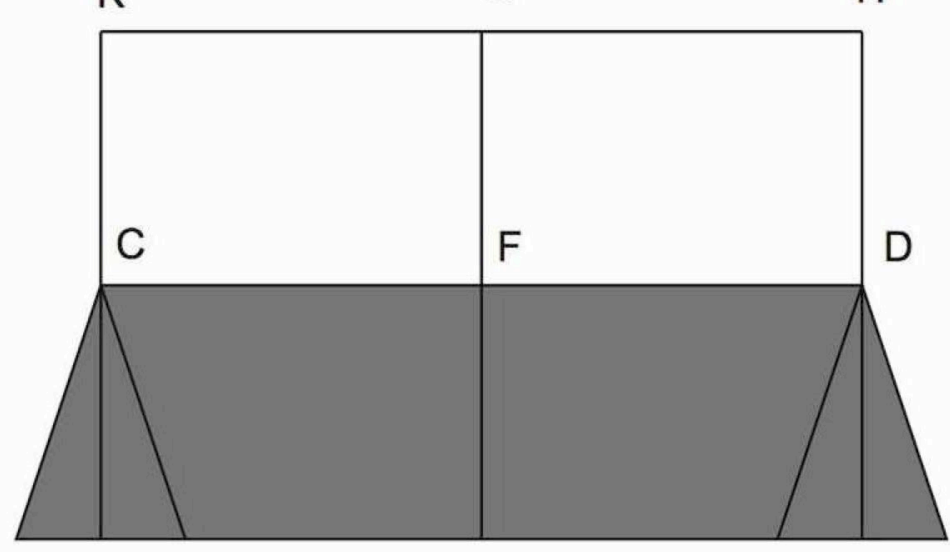

K A K

Figure 5. Summary of Khayyám's proof for the parallel postulate, based on Kanani, ${ }^{42}$ p. 317 , fig. 5 ; p. 318 , fig. 6 )

terms such as 'point', 'line' and 'plane' are undefined. ${ }^{c}$ David Hilbert once famously remarked that in a system of geometrical axioms one must be able to substitute 'point', 'line' and 'plane' by 'table', 'chair' and 'beer mug'. The increasing formalization of mathematics as a discipline in the nineteenth century was an important first step in the distancing of geometry from its ancient historical roots, as epitomized in Hilbert's set of 20 assumptions which constituted a foundation for a modern treatment of Euclidean geometry. Although this program proved untenable in the face of Gödel's incompleteness theorems, it is still maintained with some modifications. While it is impossible to formalize all of mathematics, mathematicians still aim to formalize most of it. Interestingly, though, Hilbert at the same time believed Euclidean geometry to be the closest approximation of our intuitions of space. As Majer's ${ }^{43}$ analysis of his view on geometry shows, Hilbert believed Euclidean geometry to reflect our everyday conception of space, while he regarded non-Euclidean geometries as more applicable to express

${ }^{c}$ Euclid did provide definitions for these terms, for instance, a point is defined as that which has no part. It is impossible to define every term we use, because a definition requires terms that in turn need to be defined and so on (what does it mean to have 'no part'?) - this would quickly lead to infinite regress.

scientific concepts of space-time. Indeed, the invention of non-Euclidean geometries did not automatically undermine the Kantian view of an a priori Euclidean notion of space. However, foundational approaches to geometry did lead to the growing realization that one cannot simply equate geometrical intuitions with geometry as a formal science or as a description of physical space. Poincaré's conventionalism, which holds that some mathematical theorems cannot be preferred above others on a priori intuitive grounds, serves as a good illustration. When asked which geometry was true, Poincaré ${ }^{44}$ (p. 50) famously responded:

The geometrical axioms are therefore neither synthetic a priori intuitions nor experimental facts. They are conventions [...] What then are we to think of the question: Is Euclidean geometry true? It has no meaning. We might as well ask if the metric system is true and if the old weights and measures are false [...] One geometry cannot be more true than another: it can only be more convenient.

More decisively, in the early decades of the twentieth century, Einstein's theory of relativity showed that physical time-space could be more adequately captured by non-Euclidean (Riemannian) geometry than by Euclidean geometry. Yet even then the Kantian notion of intuitive Euclidean geometry was not immediately abandoned. In his doctoral dissertation of 1922, Rudolf Carnap attempted to resolve the conflicts in foundational geometry by stating that intuitive space, geometry and physical space in fact referred to different types of space and that there was thus no contradiction. His view was strongly influenced by Husserl's concept of 'essential insight' (Wesenserschauung), which is directed at universal features of space, rather than at particular geometrical properties of the environment. For Carnap, intuitive space was synthetic a priori and experience-constituting in Kant's original sense. However, in later writings he progressively abandoned this notion (Friedman ${ }^{45}$ ). In the course of the twentieth century, Kant's argument from geometry has fallen from favor. The intellectual climates of foundational mathematics and theoretical advances in physics have together led to an increasing divergence between our intuitive notions of space and formal geometry. The same happened in other sciences, such as physics and biology which diverged increasingly from their evolved counterparts, such as intuitive physics and folk biology. Newtonian physics denied the notion of impetus, an important principle of intuitive physics, while Darwinian evolutionary theory made untenable the notion that species possess an unchanging essence, a central belief in intuitive biology (De Cruz and De 
Smedt $\left.{ }^{46}\right)$. Up to the eighteenth century, intuitions of space did play an important role in the development of geometry. The metatheoretical research programs in nineteenth century mathematics, which explicitly ignored any semantic content of mathematical objects, made non-intuitive ideas such as non-Euclidean geometries acceptable. Although non-Euclidean geometries may be better suited to describe the spatial relationships within space-time than Euclidean geometry, natural selection does not take observations on such a grand scale into account, because they are unimportant for an animal's day to day survival and reproduction. It is therefore not unlikely that natural selection could have produced Euclidean spatial intuitions in the nervous systems of humans and other animals. Since physical space and intuitions of space do not need to coincide, the argument from geometry can become an empirical question that cognitive science can address.

\section{Concluding remarks}

Kant's argument from geometry has often been interpreted as the farreaching claim that geometry reflects eternal, unchanging and universal intuitions of space. Here I have argued for a weaker interpretation of this argument, which states (1) that humans have innate, evolved and speciesuniversal cognitive adaptations to deal with space, and (2) that these intuitions constrain and govern the development of formal geometry. Geometry as a science has its roots in problems of land surveying, engineering, navigation and architecture, which require a formal solution to represent spatial relationships. Were it not for our intuitive ability to represent spatial relationships, it seems unlikely that geometry would have arisen in the first place. In this paper, I have reviewed studies that indicate that human spatial cognition emerges early in development, that it is continuous with that of nonhuman primates, and that it is subserved by specialized neural circuitry that we share with other mammals. Further empirical investigations are needed to evaluate the second part of the enhanced argument from geometry. More cross-cultural experiments are needed to test whether principles of Euclidean geometry, such as parallelism and right angles, are a stable and universal feature of human cognition. It is interesting to consider Kant's following remarks in the light of the evidence from cognitive science:

We can [...] speak of space, extended beings, and so on, only from the human standpoint. If we depart from the subjective condition under which alone we can acquire outer intuition $[. .$.$] then the$ representation of space signifies nothing at all (Kant, ${ }^{3}$ A26/B42).
Our expositions accordingly teach the reality (i.e., objective validity) of space in regard to everything that can come before us externally as an object, but at the same time the ideality of space in regard to things when they are considered in themselves through reason, i.e., without taking account of the constitution of our sensibility (Kant, ${ }^{3}$ A28/B44).

Kant argues here that it is only from our human standpoint that we can speak of space: although it exists independently from our experience, we cannot cognize space as it is in itself, but only through our a priori intuitions. Like other animals, humans can only intuitively think about space through the lens of cognitive adaptations. We can only overcome these intuitions through a rigorous formalization of geometry as a science, which as a consequence becomes less intelligible to us.

\section{Bibliography}

1. Plato, Meno, in Exploring philosophy. An introductory anthology, ed. S. M. Cahn (Oxford University Press, New York, 2000) pp. 117-51. Originally dating from ca. 380 B.C.

2. R. Descartes, Le discours de la méthode, la dioptrique, les météores et la géométrie, in Oeuvres philosophiques de Descartes, ed. F. Alquié (Classiques Garnier, Paris, 1988) pp. 549-761. Originally dating from 1637.

3. I. Kant, Critique of pure reason, ed. and transl. P. Guyer and A. W. Wood (Cambridge University Press, Cambridge, 2005). Originally dating from 1781

4. R. Hersh, What is mathematics, really? (Oxford University Press, Oxford, 1997).

5. L. Shabel, Kant's "argument from geometry", Journal of the History of Philosophy 42, 195-215 (2004).

6. L. Shabel, Reflections on Kants concept (and intuition) of space, Studies in the History and Philosophy of Science 34, 45-57 (2003).

7. H. De Cruz, Why are some numerical concepts more successful than others? An evolutionary perspective on the history of number concepts, Evolution and Human Behavior 27, 306-23 (2006).

8. L. Decock, A physicalist reinterpretion of phenomenal spaces, Phenomenology and the Cognitive Sciences 5, 197-225 (2006).

9. E. Margolis and S. Laurence, The ontology of concepts-Abstract objects or mental representations?, Noûs 41, 561-93 (2007).

10. W. V. O. Quine, Epistemology naturalized, in Ontological relativity and other essays, ed. W. V. O. Quine (Columbia University Press, New York, 1969) pp. 69-90.

11. C. V. Mora, M. Davison, J. M. Wild and M. W. Walker, Magnetoreception and its trigeminal mediation in the homing pigeon, Nature 431, 508-11 (2004). 
12. S. T. Emlen, The development of migratory orientation in young indigo buntings, Living Bird 8, 113-26 (1969).

13. D. Jackson, M. Holcombe and F. L. W. Ratnieks, Trail geometry gives polarity to ant foraging networks, Nature 432, 907-9 (2004).

14. S. P. D. Judd and T. S. Collett, Multiple stored views and landmark guidance in ants, Nature 329, 710-4 (1998).

15. A. C. Kamil and J. E. Jones, The seed-storing corvid Clarks nutcracker learns geometric relationships among landmarks, Nature 390, 277-9 (1997).

16. A. C. Kamil and J. E. Jones, How do they, indeed? A reply to Biegler et al., Animal Behaviour 57, F9-10 (1999).

17. K. L. Gould-Beierle and A. C. Kamil, The effect of proximity on landmark use in Clarks nutcrackers, Animal Behaviour 58, 477-88 (1999).

18. K. Cheng, A purely geometric module in the rats spatial representation, Cognition 23, 149-78 (1986).

19. C. R. Gallistel, The organization of learning (MIT Press, Cambridge MA, 1990).

20. K. Cheng and N. S. Newcombe, Is there a geometric module for spatial orientation? Squaring theory and evidence, Psychonomic Bulletin and Review 12, 1-23 (2005).

21. G. Vallortigara, M. Zanforlin and G. Pasti, Geometric modules in animals' spatial representations: A test with chicks (Gallus gallus domesticus), Journal of Comparative Psychology 104, 248-54 (1990).

22. J. P. Vargas, J. C. López, C. Salas and C. Thinus-Blanc, Encoding of geometric and featural information by goldfish (Carassius auratus), Journal of Comparative Psychology 118, 206-16 (2004).

23. L. B. Day, N. Ismail and W. Wilczynski, Use of position and feature cues in discrimination learning by the whiptail lizard (Cnemidophorus inornatus), Journal of Comparative Psychology 117, 440-8 (2003).

24. L. Hermer and E. S. Spelke, A geometric process for spatial reorientation in young children, Nature 370, 57-9 (1994).

25. L. Hermer and E. S. Spelke, Modularity and development: The case of spatial reorientation, Cognition 61, 195 -232 (1996).

26. L. Hermer-Vazquez, A. Moffet and P. Munkholm, Language, space, and the development of cognitive flexibility in humans: The case of two spatial memory tasks, Cognition 79, 263-99 (2001).

27. D. B. M. Haun, J. Call, G. Janzen and S. C. Levinson, Evolutionary psychology of spatial representations in the Hominidae, Current Biology 16, 1736-40 (2006).

28. B. Landau, H. Gleitman and E. S. Spelke, Spatial knowledge and geometrical representation in a child blind from birth, Science 213, 1275-8 (1981).

29. J. O'Keefe and J. Dostrovsky, The hippocampus as a spatial map. Preliminary evidence from unit activity in the freely-moving rat, Brain Research 34, 171-5 (1971).

30. C. Lever, T. Wills, F. Cacucci, N. Burgess and J. O'Keefe, Longterm plasticity in hippocampal place-cell representation of environmental geometry, Nature 416, 90-4 (2002).
31. E. Save, A. Cressant, C. Thinus-Blanc and B. Poucet, Spatial firing of hippocampal place cells in blind rats, The Journal of Neuroscience 18, 1818-26 (1998).

32. T. Hafting, M. Fyhn, S. Molden, M.-B. Moser and E. I. Moser, Microstructure of a spatial map in the entorhinal cortex, Nature 436, 801-6 (2003).

33. F. Sargolini, M. Fyhn, T. Hafting, B. L. McNaughton, M. P. Witter, M.B. Moser and E. I. Moser, The hippocampus as a spatial map. Preliminary evidence from unit activity in the freely-moving rat, Science 312,758-62 (2006).

34. M. P. Witter and E. I. Moser, Spatial representation and the architecture of the entorhinal cortex Trends in Cognitive Sciences 29, 671-8 (2005).

35. A. D. Ekstrom, M. J. Kahana, J. B. Caplan, T. A. Fields, E. A. Isham, E. L. Newman and I. Fried, Cellular networks underlying human spatial navigation, Nature 425, 184-7 (2003).

36. R. Epstein and N. Kanwisher, A cortical representation of the local visual environment, Nature 392, 598-601 (1998).

37. R. Epstein, E. A. DeYoe, D. Z. Press, A. C. Rosen and N. Kanwisher, Neuropsychological evidence for a topographical learning mechanism in parahippocampal context, Cognitive Neuropsychology 18, 481-508 (2001)

38. D. B. M. Haun, C. J. Rapold, J. Call, G. Janzen and S. C. Levinson, Cognitive cladistics and cultural override in hominid spatial cognition, Proceedings of the National Academy of Sciences of the United States of America 103, 17568-73 (2006)

39. S. Dehaene, V. Izard, P. Pica and E. S. Spelke, Core knowledge of geometry in an Amazonian indigene group, Science 311, 311-84 (2006).

40. S. Atran, Folk biology and the anthropology of science: Cognitive universals and cultural particulars, Behavioral and Brain Sciences 21, 547-69 (1998).

41. T. Callaghan, P. Rochat, A. Lillard, M. L. Claux, H. Odden, S. Itakura S. Tapanya and S. Singh, Synchrony in the onset of mental-state reasoning evidence from five cultures, Psychological Science 16, 378-84 (2005).

42. N. Kanani, Omar Khayyám and the parallel postulate, Animal Behaviour 8, 309-26 (2004).

43. U. Majer, Geometry, intuition and experience: From Kant to Husserl, Erken ntnis 42, 261-85 (1995).

44. H. Poincaré, Science and hypothesis (Walter Scott Publishing, London, 1905).

45. M. Friedman, Carnap and Weyl on the foundations of geometry and relativity theory, Erkenntnis 42, 247-60 (1995).

46. H. De Cruz and J. De Smedt, The role of intuitive ontologies in scientific understanding - The case of human evolution, Biology and Philosophy 22, 351-68 (2007).

\section{Acknowledgements}

I gratefully acknowledge all the useful comments given at the occasion of my presentation at PMP2007, especially those by Jean Paul Van Bendegem, Jens Høyrup, Madeline Muntersbjorn, Yehuda Rav, José Ferreirós, and Ed- 
uard Glas. Special thanks to Johan De Smedt, Pierre Pica and two anonymous reviewers for their helpful suggestions. This research was supported by grant OZR916BOF from the Free University of Brussels. 